\title{
Influencia de un programa de promoción de la salud en población laboral
}

\author{
Influence of a program for the promotion \\ of health in workers
}

Robinson Ramírez-Vélez, FT, PhD@*,

Luís F. Lastra, LIC ${ }^{* *}$,

Ricardo A. Agredo-Zuñiga, FT, MG@ ${ }^{* * *}$,

Carlos A. López-Alban, MD, MSc****

Fecha de Recepción: Septiembre 9 de 2009

Fecha de Aceptación: octubre 16 de 2009

\section{Resumen}

El Objetivo de la Investigación fue evaluar la influencia de un programa de promoción de la salud sobre la calidad de vida, la capacidad funcional y la composición corporal, en una población laboral. Se utilizó un método de estudio cuasi-experimental en el que participaron en 62 sujetos masculinos, mayores de 18 años, saludables, intervenidos en un programa de cambio de comportamientos con ejercicio físico y nutrición saludable por 16 semanas. Los cambios en la calidad de vida (Cuestionario de Salud SF-12), capacidad funcional ( $\left.V O_{2 \max }\right)$, y la composición corporal (Antropometría) fueron los indicadores de resultado. Luego de la intervención, se encontraron cambios significativos en indicadores antropométricos y funcionales: Perímetro abdominal ( $p<0,01)$, perímetro de cadera $(p<0,05)$, respuesta cardiovascular al ejercicio ( $p<0,01)$, capacidad funcional y flexibilidad $(p<0,05)$. No se encontraron diferencias en el resultado de calidad de vida. Este trabajo, demostró los beneficios clásicos del ejercicio físico y los cambios en los patrones y conductas alimentarias, sobre la composición corporal y la capacidad funcional, señalando la efectividad de la implementación de programas de promoción de la salud y cómo estos cambios de estilos de vida minimizan o reducen los factores de riesgo asociados a enfermedades crónicas, las cuales conducen a discapacidad laboral.

Palabras clave: Ejercicio físico, calidad de vida, composición corporal, capacidad funcional,

\footnotetext{
* Fundación Universitaria María Cano, Extensión Cali, Colombia. robin640@ hotmail.com

** Centro de Acondicionamiento Físico y Rehabilitación en Lesiones Deportivas Megalastra. Cali, Colombia. profe2005@yahoo.com

****Fundación Universitaria María Cano, Extensión Cali, Colombia. fitsaludintegral@ gmail.com

****Centro para la Investigación en Salud y Rendimiento Humano ZOE Calidad de Vida. Cali, Colombia. fitvalle@ hotmail.com
}

|REV.COL. REH. | Bogotá, Colombia | Vol. 8 | No. 1 |pg. 67 - 76 | octubre | 2009 | ISSN. 1692-1879| 


\section{Abstract}

The Investigation Blank was assessment the influence of program on the quality of life, functional capacity and body composition, in population of workers. Methods used was cuasi-experimental analytic in 62 subject's male, > 18 years, healthy, intervened in a program of behaviour change with physical activity and healthy nutrition for 16 weeks. Changes in the quality of life (Health Questionnaire SF-12), functional capacity $\left(\mathrm{VO}_{2 \max }\right)$, and body composition (anthropometry) were indicators of outcome. Following the intervention were significant changes in anthropometric indicators and functional abdominal perimeter $p<0.01$, perimeter hip $p<0.05$, cardiovascular response to exercise $p<0.01$, functional ability and flexibility $p<0.05$. There was no difference in the outcome of quality of life. This work in a working environment unique in our system of public health, showed the classic benefits of physical exercise and changes in dietary patterns and behaviours on the body composition and functional capacity, confirming that the implementation of prevention programmes and changes in lifestyles minimize the risk of chronic diseases which lead to worker's disability.

Key Words: Fitness, quality of life, body composition, capacity functional.

\section{Introducción}

La relación entre la percepción de la salud o Calidad de Vida Relacionada con la Salud (CVRS), la capacidad funcional $\left(\mathrm{VO}_{2 \max }\right)$ y algunos indicadores antropométricos como la obesidad abdominal y el Índice de Masa Corporal (CDC Atlanta 1996)(1) han sido asociados con mayor riesgo de morir por múltiples causas, especialmente por enfermedades relacionadas con el sistema cardiovascular (2). Se postula que la aparición de enfermedades crónicas se debe a factores de riesgo muchos de ellos prevenibles (3), destacándose la hipertensión arterial (HTA), la diabetes mellitus (DM), la obesidad, la intolerancia a la glucosa y la dislipidemia. Varios estudios han mostrado la concomitancia entre una baja capacidad física y la aparición de enfermedades cardiovasculares (5)(4). Por lo tanto, la evaluación de un individuo desde una perspectiva integral, se convierte en una estrategia de detección temprana para poner en marcha medidas preventivas y correctivas que pueden ser implementadas desde el modelo de promoción de la salud.

Debido a que el nivel de capacidad física y el empleo son aspectos importantes de la vida adulta, se podría asumir que las medidas de capacidad funcional por $\mathrm{VO}_{2 \max }$ deberían correlacionarse positivamente con las medidas de Calidad de Vida Relacionada con la Salud (CVRS); sin embargo los datos disponibles en la literatura son escasos. Boer et al (6) realizaron una intervención con el objetivo de modificar el estilo de vida en un entorno laboral. Los resultados demostraron que el Índice de Carga Laboral (Evaluado con el ICL) era menor en el grupo de sujetos que habían sido intervenidos, y que esté se asociaba con mejores puntuaciones en la CVRS (Evaluada con SF-36). Así mismo, Chiu et al. (7) evaluaron la relación entre la CVRS y el ICL en 2173 sujetos (Con edades comprendidas entre 20-67 años) en un entorno hospitalario y de producción de sectores públicos y privados de Taiwán. Los resultados mostraron diferencias y correlaciones estadísticas significativas en los dominios físicos y mentales del cuestionario de CVRS elaborado por la OMS, en los sujetos que reportaban una mejor capacidad física (8).

Adicionalmente, diferentes autores, han reportado los efectos protectores de la práctica de actividad física regular y una composición corporal 
saludable (Medida por indicadores antropométricos) frente a la aparición y riesgo de padecer Enfermedades Crónicas No Transmisibles (ECNT), como Enfermedad Coronaria, Hipertensión, Accidente Cerebrovascular, Diabetes Mellitus, Osteoporosis, Depresión y Ansiedad (9, 10). En relación con esto, un aumento de la actividad física y capacidad funcional por $\mathrm{VO}_{2 \max }$ se asocia a una mejor CVRS en adultos $(11,12)$. Sin embargo, un trabajo realizado por Smolander et al. (13), mostró que no había relación con los cambios en los dominios de la CVRS y el aumento de la actividad física y la capacidad física. Contrario a lo encontrado en un programa de ejercicio físico llevado a cabo en entornos de trabajo, donde se demostró incrementos de la capacidad física e impidió la rápida disminución de la CVRS en trabajadores de cuidados en el hogar $(14,15)$. Razonamientos teóricos, podrían explicar que una composición corporal ideal y una capacidad funcional adecuada, puede aumentar en un individuo sano la capacidad para hacer frente a las exigencias de la vida cotidiana, pero las vías para la mejoría global de la capacidad funcional o de la CVRS pueden ser muy confusas debido a muchos factores que influyen en estos conceptos. Por ello, organismos internacionales recomiendan el fomento de estilos de vida saludables, como una importante medida de promoción de la salud y prevención de la discapacidad. Por ejemplo, la Organización Mundial de la Salud (OMS), ha emprendido en las últimas décadas programas para reducir factores de riesgo asociados a síndrome metabólico y enfermedades cardiovasculares que estimulen el auto-cuidado en la población empresarial (16). Estos programas se basan en la realización regular de actividad física, cambios en los patrones alimenticios, espacios libres de humo, pausas activas, entre otros, los cuales proporcionan una mejoría en la percepción de la salud o CVRS, debido a los beneficios que estos ofrecen.

Así mismo, estudios epidemiológicos han sido realizados para identificar y evaluar intervenciones sobre factores de riesgo en la población general (17,
18). Sin embargo, pocos se han enfocado hacia la población trabajadora, factor humano de primordial importancia quienes además de encontrarse sometidos a factores de riesgo laboral, pueden presentar factores de riesgos de enfermedades crónicas asociadas al estilo de vida, que aunados a los primeros conducen al incremento de discapacidad laboral (19). Por tanto, evaluar intervenciones a nivel colectivo desde una perspectiva integral, se convierte en una estrategia que se reflejaría directamente en la productividad económica y social de una empresa. Este trabajo se ha centrado en evaluar la influencia de un programa de promoción de estilos de vida saludable sobre la calidad de vida, la capacidad funcional y la composición corporal de trabajadores de mediana edad, esperando que sirva como referente para la implementación de modelos de salud integral, dentro del contexto de "Empresa Saludable".

\section{Método}

Se utilizó un diseño cuasi-experimental analítico. Los participantes fueron empleados bancarios, del área administrativa y jurídica que cumplían jornadas laborales de 8 horas, frente al computador, del área metropolitana de la ciudad de Cali, Colombia. Se convocaron 115 trabajadores de los cuales $n=62$ individuos cumplieron los criterios de inclusión (género masculino, mayores de 18 años, aparentemente saludables y aceptación al estudio mediante la firma de consentimiento informado). Se excluyeron sujetos que presentaran cualquiera de las siguientes patologías por antecedentes médicos y personales: diagnóstico previo de DM tipo 1 ó 2 , enfermedad crónica o inflamatoria, procesos infecciosos de cualquier etiología agudos o crónicos, presencia de cualquier enfermedad que afecte el metabolismo de la glucosa o los lípidos, rechazo a firmar el consentimiento informado, o condición mental que afectará la autonomía del participante.

Técnicas e Instrumentos. La información se recolectó Pre y post intervención mediante entrevista personal, aplicación de encuesta sociodemográfica y examen físico que incluyó: antropometría, usando 
el protocolo de Estrada et al. 1998 (20) y López et al. (21). Se registró la talla con equipo Kramer® y peso corporal con balanza Tanita ${ }^{\circledR}$. Con estas variables se calculó el Índice de masa corporal (IMC) en $\mathrm{kg} / \mathrm{m} 2$ y se consideró normal entre 18.5 y 24.9; sobrepeso de 25.0 a 29.9 ; y obesidad $>30 \mathrm{~kg} / \mathrm{m} 2$. Adicionalmente, con cinta métrica se midió el perímetro de la cintura y cadera $(\mathrm{cm})$ siguiendo la referencia de Brook RD et al. (22), considerando factor de riesgo de Enfermedades Cardiovasculares $(\mathrm{ECV})$ perímetro de cintura $=88.1 \mathrm{~cm}$. La capacidad funcional fue valorada mediante prueba ergométrica en cicloergómetro Monark, tipo Ergometric 828E para determinar el calculo indirecto del VO2max en $(\mathrm{mL} / \mathrm{kg} / \mathrm{min})$ y la clasificación de la capacidad funcional en METs, se utilizó como estimador de riesgo de enfermedades crónicas. La frecuencia cardíaca (FC) fue monitorizada electrónicamente con pulsómetro Polar Inc A-5 desde el inicio hasta el final de la prueba (21). La flexibilidad general fue evaluada mediante la prueba de Wells (21). La calidad de vida relacionada con la salud (CVRS), se evaluó a través del Cuestionario Genérico de Salud, 12-items short form the SF-12® Health Survey validado por Lugo (23) versión reducida del SF-36; que recoge medidas sobre el estado funcional PCS-(Componente Físico) y emocional MCS-(Componente Mental).

Intervención. Los autores del estudio, diseñaron un programa de promoción de la salud (como técnica) para evaluar la efectividad de la modificación de patrones y conductas de estilo de vida en el marco de "Empresa Saludable", que incluía la práctica regular de ejercicio físico y modificación de conductas nutricionales, orientados y dirigidos por un equipo biomédico (Medico Deportólogo, Nutricionista, Educadores Físicos y Fisioterapeutas) con amplia experiencia en el tema. La actividad física se desarrolló con una frecuencia de 3 veces por semana ( 2 sesiones de gimnasia dirigida y reforzamiento muscular y 1 sesión en campo deportivo-Fútbol), una hora por sesión durante un periodo de 16 semanas. Los programas de ejercicio aeróbico, se realizaron a una intensidad entre el $50 \%$ y $65 \%$ de la $F C_{\max }$, alcanzada en la prueba de capacidad física. Protocolo: $10 \mathrm{mi}-$ nutos de calentamiento, 30 minutos de ejercicio aeróbico, 5 minutos de enfriamiento y estiramiento. Dicho protocolo se asentó en los lineamientos y guías internacionales para la prescripción de ejercicio del American College Sport Medicine (24).

Aspectos éticos. El protocolo fue presentado a la Junta Administrativa y al Departamento de Medicina Laboral y Preventiva ARP Seguros ALFA SA, amparado en las disposiciones vigentes para la protección de los sujetos humanos que participan en investigación contenidas en la resolución 8430 de 1993 del Ministerio de Salud. Antes de ser incluidos los participantes recibieron explicación sobre la evaluación médica y fisioterapéutica que se les realizaría para el conocimiento del estado y percepción de su salud, así como de la intervención y firma del consentimiento informado.

Análisis Estadístico. Los resultados de la intervención se procesaron en el programa SPSS versión 11.5( ) mediante estadísticos descriptivos. Las diferencias pre y post-intervención fueron testeadas con la prueba $t$ student para muestras relacionadas. El coeficiente de correlación de Pearson (r), se utilizó para conocer la relación y cambios entre las variables capacidad funcional, indicadores antropométricos y el SF12 , en sus componentes físico, mental y las categorías internas del instrumento. Un valor $\mathrm{p}<0,05$ se consideró como significante.

\section{Resultados}

De los 62 adultos saludables incluidos al inicio del estudio, $10 \%$ (correspondiente a 6 sujetos) no terminaron con la intervención por motivos ajenos al estudio ( 2 individuos por cambio en las jornadas laborales, 3 sujetos por modificaciones de cargos administrativos y 1 por disponibilidad de tiempo). En la Tabla 1, se presentan los resultados de los indicadores antropométricos de las 62 personas que asistieron al programa en las 16 semanas. Cambios significativos fueron encontrados en las variables perímetro abdominal $(13 \%) \mathrm{p}<0,01$ y perímetro de cadera $(3 \%) \mathrm{p}<0,05$. La tabla 2 , reúne los cambios 
luego de la intervención en las variables respuesta cardiovascular al ejercicio $\mathrm{p}<0,01$, capacidad funcional y flexibilidad $\mathrm{p}<0,05$. No se encontraron diferencias en los resultados de las puntuaciones del Cuestionario SF-12 entre las escalas normalizadas física y mental.

El análisis de correlación en los dos componentes del SF-12 y las categorías internas del instrumento, destaca los cambios en esfuerzos moderados y el PCS-12 r=0,69 p<0,01 y Desanimado con el PCS-12 r=,34 p<0,01 en el compo- nente físico. También correlaciones entre la Salud general y el MCS-12 r=,34 p<0,01; Desanimado y MCS-12 r=,79 p<0,01 en el componente mental, tabla 3. Igualmente, se encontraron correlaciones en las variables Peso y Grasa Corporal, pre y post intervención, $\mathrm{r}^{2}=0,59, \mathrm{r}^{2}=0,49$ respectivamente, Figura 1A, p<0,01, Peso Corporal y Perímetro Abdominal $r^{2}=0,69$ (Pre-Intervención), $r^{2}=0,67$ (Pos-Intervención), Figura 1B, p<0,01. Por último, se resalta el efecto del programa en las correlaciones de la composición corporal (IMC) y $\mathrm{VO}_{2 \max }$ $\mathrm{r}^{2}=0,15$ (Pre- Intervención) a $\mathrm{r}^{2}=0,24$ (Pos-Intervención), respectivamente, demostrado en la Figura 1.

TABla 1

INDICADORES ANTROPOMÉTRICOS DE LA POBLACIÓN ESTUDIADA

\begin{tabular}{lcc}
\hline Variables & Preintervención & Posintervención \\
Media $(\mathbf{D E})$ & & \\
\hline Peso $(\mathrm{kg})$ & $73,9 \pm 9,5$ & $72,3 \pm 8,4$ \\
Talla $(\mathrm{m})$ & $1,70 \pm 5,0$ & $1,70 \pm 4,9$ \\
$\mathrm{IMC}\left(\mathrm{kg} / \mathrm{m}^{2}\right)$ & $25,3 \pm 2,8$ & $24,7 \pm 2,4$ \\
$\%$ de grasa & $17,4 \pm 2,9$ & $17,4 \pm 2,6$ \\
Perímetro abdominal $(\mathrm{cm})$ & $83,1 \pm 7,8$ & $72,3 \pm 8,4 * *$ \\
Perímetro de cadera $(\mathrm{cm})$ & $97,4 \pm 57,0$ & $94,9 \pm 5,2 *$ \\
ICC & $\mathrm{s} 0,86 \pm 0,07$ & $0,81 \pm, 001$ \\
Desviación estándar (DE). *p<0,05;**p<0,01 & \\
ICC (Índice cintura cadera), IMC (Índice de masa corporal) & \\
\hline
\end{tabular}

TABla 2

DESCRIPCIÓN DE LA CAPACIDAD FÍSICA, RESPUESTA CARDIOVASCULAR AL EJERCICIO Y CALIDAD DE VIDA

\begin{tabular}{lcc}
\hline Variables & $\begin{array}{c}\text { Preintervención } \\
\text { Media }(\mathbf{D E})\end{array}$ & Posintervención \\
\hline FC reposo $(\mathrm{lpm})$ & $78,4 \pm 12,9$ & $70,0 \pm 0,6 * *$ \\
FC máx. $(\mathrm{lpm})$ & $173,4 \pm 11,3$ & $180,1 \pm 11,5 * *$ \\
$\mathrm{VO}_{2 \max }(\mathrm{mL} / \mathrm{kg} / \mathrm{min})$ & $40,7 \pm 5,6$ & $42,5 \pm 4,8^{*}$ \\
Mets & $11,6 \pm 1,7$ & $12,1 \pm 1,3 *$ \\
Test de Wells $(\mathrm{cm})$ & $17,6 \pm 9,8$ & $28,2 \pm 7,3 *$ \\
PCS-12 & $44,5 \pm 3,1$ & $44,3 \pm 2,8$ \\
MCS-12 & $47,5 \pm 3,9$ & $48,3 \pm 3,4$ \\
Desviación estándar $(\mathrm{DE}) . * \mathrm{p}<0,05 ; * * \mathrm{p}<0,01$ & \\
\hline
\end{tabular}


TABLA 3

Correlaciones entre las Categorías internas del Cuestionario 12-ITEm Short-Form health SURVEY (SF-12) y los Componentes Físicos (PCS-12) y Mentales (MCS-12).

\begin{tabular}{|c|c|c|c|c|}
\hline Categorías internas & \multicolumn{2}{|c|}{$\begin{array}{l}\text { PCS-12a } \\
\text { MCS-12 }\end{array}$} & \multicolumn{2}{|c|}{$\begin{array}{l}\text { PCS-12 } \\
\text { MCS-12 }^{b}\end{array}$} \\
\hline 1. Salud en general & $0,36 * *$ & 0,16 & $-0,10$ & $-0,34 * *$ \\
\hline 2. Esfuerzos moderados & $0,46 * *$ & $0,69 * *$ & $-0,39 * *$ & $-0,20$ \\
\hline 3. Subir varios pisos & 0,05 & $0,37 * *$ & 0,14 & $-0,01$ \\
\hline 4. Hacer menos de lo deseado & 0,07 & 0,09 & $-0,09$ & 0,07 \\
\hline 5. Dejar tareas & $0,30 * *$ & $-0,26^{*}$ & 0,14 & 0,21 \\
\hline 6. Menos de lo deseado por emociones & $-0,05$ & $-0,08$ & $0,38 * *$ & 0,23 \\
\hline 7. No tan cuidadoso por emociones & $-0,37 * *$ & 0,09 & $0,51 * *$ & $0,27 *$ \\
\hline 8. El dolor dificulta trabajo & $0,47 * *$ & $0,41 * *$ & 0,12 & 0,12 \\
\hline 9. Calmado & $-0,02$ & $-0,01$ & $0,55 * *$ & $0,56 * *$ \\
\hline 10. Energía & 0,20 & $-0,02$ & $0,37 * *$ & $0,33 * *$ \\
\hline 11. Desanimado & $-0,25^{*}$ & $-0,34 * *$ & $0,55 * *$ & $0,79 * *$ \\
\hline 12. Función social - frecuencia & $-0,02$ & $-0,09$ & $0,39 * *$ & $0,31 *$ \\
\hline
\end{tabular}

a Preintervención; ${ }^{\mathbf{b}}$ Posintervención. *p<0,05; **p<0,01, coeficiente de correlación de Pearson, Physical Component Summary (PCS-12); Mental Component Summary (MCS-12)

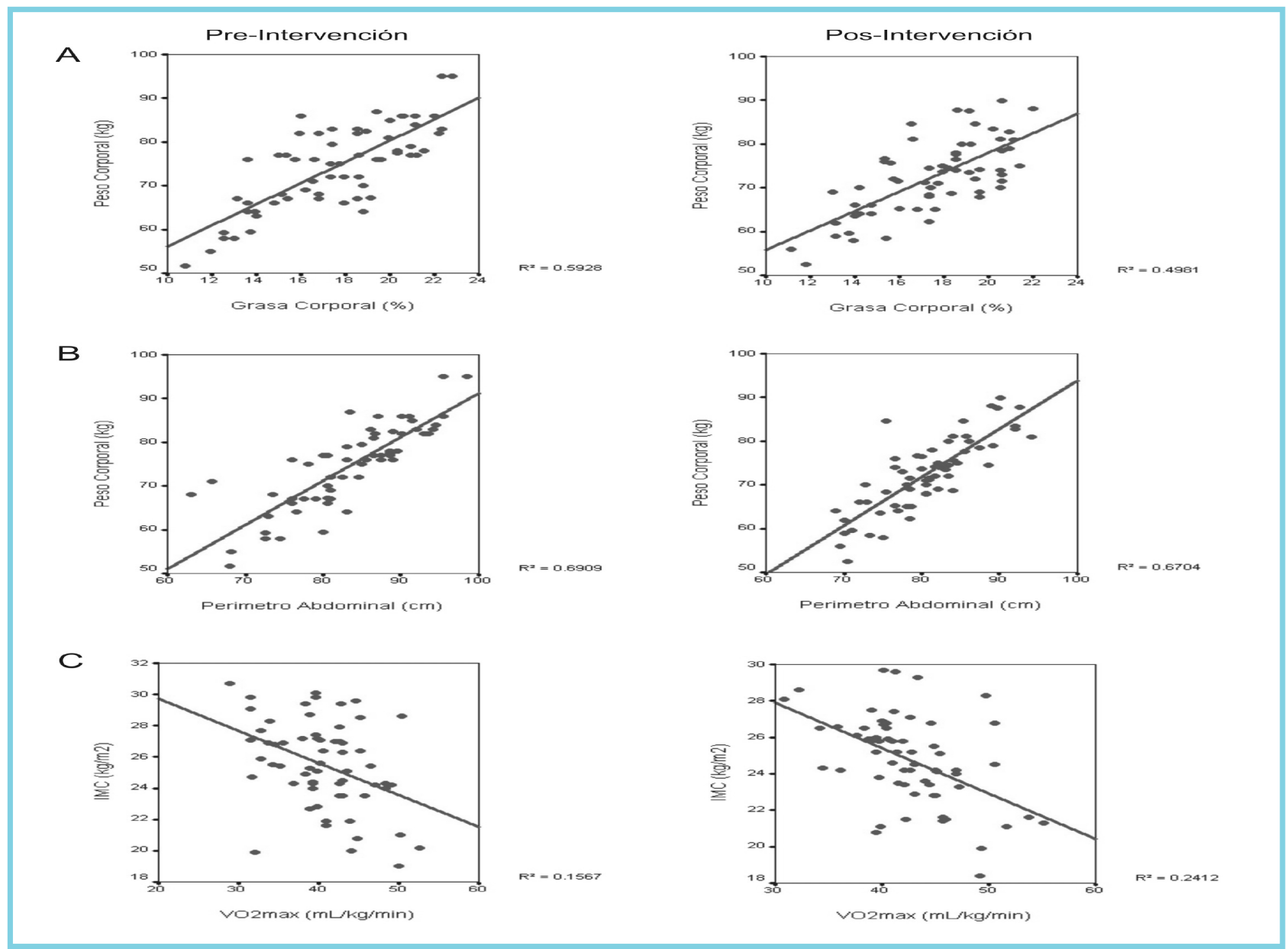

Figura 1. Correlaciones entre indicadores antropométricos y funcionales pre y posintervención. 


\section{Discusión}

El objetivo principal de este estudio fue evaluar la influencia de un programa de promoción de estilos de vida saludable sobre la calidad de vida, la capacidad funcional y la composición corporal en un grupo de trabajadores de mediana edad en un entorno laboral. El SF-12 es uno de los métodos de encuesta mas frecuentemente utilizados para evaluar la CVRS, tanto en investigación social como en la práctica clínica. Este estudio en trabajadores, es el primero en su género que explora las dimensiones internas de este instrumento con los indicadores de salud asociados a enfermedades crónicas (composición corporal y capacidad física) en una intervención integral.

Suficiente evidencia apoya los efectos sinérgicos y protectores de la práctica regular de actividad física y una composición corporal saludable, sobre la aparición de Enfermedades Crónicas No Transmisibles (ECNT) como la Enfermedad Coronaria, Hipertensión Arterial, Accidente Cerebrovascular, Diabetes Mellitus, Osteoporosis, Depresión y Ansiedad $(9,10)$. A la vista de los resultados, resulta sorprendente la mejora obtenida en la condición física teniendo en cuenta la corta duración del programa (16 semanas), confirmando el tópico general que recomienda un mínimo de tres días a la semana para la mejora de los parámetros de condición física y salud propuestos por el (American College of Sports Medicine). Se observa que tres sesiones semanales con una intensidad adecuada pueden producir efectos beneficiosos, lo que concuerda con otros estudios utilizados como referencia $(1,2,3,4$, 5). Del mismo modo hay que reseñar, que todas las variables se encuentran dentro de la normalidad en comparación con los valores normativos de referencia internacionales considerados como saludables $(7,8)$.

Por lo que respecta a la valoración de la composición corporal, a través del perímetro abdominal, se produce una diferencia estadística significante, $\mathrm{p}<0,01$, mostrando que la activi- dad física es muy importante para reducir los niveles de peso graso a corto plazo, lo cual concuerda con otros trabajos internacionales (13). Este parámetro tiene una relevancia importante, ya que ha sido propuesto recientemente como un índice más predictivo del riesgo de enfermedad cardiovascular que el índice de masa corporal $(1,2,3,4,5)$.

En lo referente al efecto de la actividad física sobre la CVRS en adultos con o sin riesgo cardiovascular, los resultados en la actualidad todavía son confusos (25). Por ejemplo, Smolander et al. (13), describió que no hay relación con los cambios en las categorías de la CVRS, con el aumento de la actividad física y/o capacidad funcional por $\mathrm{VO}_{2 \max }$, resultados similares a los encontrados en este estudio. Por el contrario, resultados de intervenciones con ejercicio físico llevado a cabo en trabajadores han mostrado incrementos en el $\mathrm{VO}_{2 \max }$ a medida que la CVRS mejora $(12,13)$. Estas diferencias podrían ser explicadas por el diseño de los estudios y el tiempo de las intervenciones que generalmente son mayores a 6 meses. Este estudio comprobó que a pesar de no haber cambios estadísticamente significativos en las categorías del SF-12, si hay modificaciones importantes en las variables internas del cuestionario (tanto en el componente físico como en el mental), luego de 16 semanas con ejercicio físico y hábitos alimenticios, tabla 3.

Cieza y Stucki en el 2005 (26), compararon el contenido de los diferentes instrumentos de CVRS propuestos por la OMS y la Clasificación Internacional del Funcionamiento, Discapacidad y Salud (CIF). Los resultados mostraron grandes diferencias del cuestionario SF-36 y los instrumentos para evaluar CVRS, sobre todo en temas directamente relacionados con el rendimiento físico como correr, hacer ejercicio, transportarse, subir escaleras, caminar cortas y largas distancias, en población de trabajadores.

En comparación con los estudios de referencia para la realización de este trabajo (Taiwán y Finlandia), hombres de edad similar, presentaron pun- 
tuaciones mas altas en las categorías de Dolor Corporal y Salud Física $(27,28)$. Esto podría ser explicado porque la muestra estudiada en los referentes internacionales están en entornos más exigentes físicamente (construcción, restaurantes, personal medico) y sin intervenciones, a diferencia de la población evaluada en este estudio. Otras interacciones entre la vida laboral y familiar pueden ser determinantes importantes para modificar la CVRS y en lo que respecta a la percepción de la salud, capacidad física y el bienestar (29). Estudios anteriores también han informado de efectos en la modificación de las actividades de tiempo libre y la satisfacción con la vida, evaluada con el SF-36 $(6,3,9)$ confirmando aún más la necesidad de desarrollar el concepto de promoción de la salud y fomento de actividad física y nutrición balanceada.

Se conoce que muchos problemas posturales y musculares, así como algunas lesiones, sobre todo en adultos, están relacionados con la falta de flexibilidad. La población evaluada presentó alcances en la prueba de flexibilidad de $17,6 \pm 9,8 \mathrm{~cm}$ al inicio de la intervención y luego de 16 semanas se logró un cambio significativo a $28,2 \pm 7,3 \mathrm{~cm}$., $\mathrm{p}<0,05$. Si tomamos como referencia valores internacionales se puede establecer que los sujetos evaluados en este estudio pasaron de tener regular a buena flexibilidad (30). Este hallazgo es muy importante en la salud de los trabajadores, pues se postula que un $67 \%$ de las cifras por ausentismo laboral son asociados a problemas de la parte baja (dorso-lumbar) de la espalda y son causados a un alineamiento impropio de la columna vertebral y la pelvis, como resultado directo de la disminución de la flexibilidad y debilidad muscular, beneficio fisiológico que pudo ser demostrado con la intervención.

\section{Conclusiones}

Del presente trabajo se pueden extraer las siguientes conclusiones, que deben ser interpretadas con cautela puesto que, el tamaño de la muestra y la inexistencia de grupo control así lo sugieren: 1. Un programa de estilos de vida saludable de corta duración se muestra eficaz en algunos componentes de la composición corporal y 2 . Se consiguen mejoras en la mayoría de los parámetros de la condición física relacionados con la salud en especial en el $\mathrm{VO}_{2 \max }$ y flexibilidad. Por Último, este trabajo único en su genero en nuestro sistema de salud publica demostró los beneficios clásicos del ejercicio físico que junto a una modificación en los patrones y conductas alimentarías ratifican que las empresas no deben conformarse con el hecho de ser solo creadoras de productos, servicios y del desarrollo social, sino también deben fomentar la promoción de entornos saludables a través del desarrollo de políticas internas institucionales que incluyan intervenciones integrales y educativas con sensibilización y participación activa de los trabajadores.

Agradecimientos. A la ARP SEGUROS ALFA, seccional Cali, especialmente el comité de salud ocupacional por permitir la aplicación del protocolo, a los fisioterapeutas Esther Julia Fernández y Jhon Alexis García por el apoyo técnico en la medición antropométrica y funcional.

\section{Referencias}

1. US Department of Health and Human Services. Physical activity and health: a report of the Surgeon General. Atlanta $(\mathrm{Ga})$ : US Department of Health and Human Services, Centers for Disease Control and Prevention, National Center for Chronic Disease Prevention; 1996.

2. Kaplan D, Goldberg S, Everson R, Cohen $\mathrm{R}$, Salonen J, Tuomilehto et al. Perceived health status and morbidity and mortality: evidence from the Kuopio ischaemic heart disease risk factor study. Int J Epidemiol 1996; 25: 259-65.

3. Berríos X, Koponen T, Huiguang T, Khaltaev $\mathrm{N}$, Puska P, Nissinen A. Distribution and prevalence of major risk factors of 
noncommunicable diseases in selected countries: the WHO Inter- Health Programme. Bulletin of the World Health Organization 1997;75:99-108.

4. British Cardiac Society, British Hyperlipidaemia Association, British Hypertension Society, British Diabetic Association. Joint British recommendations on prevention of coronary heart disease in clinical practice: summary. BMJ 2000;320:705-8.

5. Kannel WB. Risk stratification in hypertension: new insights from the Framingham Study. Am J Hypertens. 2000;13:3S-10S.

6 De Boer G, Van Beek J, CJ Durinck J, Verbeek F, Van Dijk. An occupational Health intervention programme for workers at risk for early retirement; A Randomised Controlled Trial, Occup. Environ. Med. 2004;61:924-929.

7. Chiu MC, Wang MJ,. Lu CW, Pan XM, Kumashiro M. The work ability index and quality of life, J. Erg. Occup. Saf. Health 2003;5:(Suppl. August); 67-69.

8. Saxena D, Carlson R, Billington. WHOQOL Group, World Health Organisation Quality Of Life, The WHO quality of life assessment instrument (WHOQOL-Bref): the importance of its items for cross-cultural research, Qual. Life. Res. 2001;10 (Suppl. 8):711-721.

9. Dunn A, Trivedi M, O’Neal H. Physical activity dose response effects on outcomes of depression and anxiety, Med. Sci. Sports Exerc. 2001;33: 5587-5597.

10. Weyer S, Kupfer B. Physical exercise and psychological health, Sports Med. 1994;17:108-116.

11. Rejeski W, Mihalko S. Physical activity and quality of life in older adults, J. Gerontol. (Ser. A) 2001;56:23-35 (Special Issue II).

12. Ramírez-Vélez R, Alban C, Reina HR, Idarraga M, Gensini FG. Beneficios percibidos de un grupo de mujeres en climaterio incorporados a un programa de actividad física terapéutica. Apunts Medicina de l'Esport. 2008;157:14-23.
13. Smolander J, Blair SN, Kohl HW. Work ability, physical activity, and cardiorespiratory fitness: 2-year results from project active, J. Occup. Environ. Med. 2000;42: 906-910.

14. Pohjonen T, Ranta R. Effects of worksite physical exercise intervention on physical fitness, perceived health status, and work ability among home care workers: five-year follow-up, Prev. Med. 2001;32: 465-475.

15. Sörensen LE, Pekkonen MM, Männikkö KH, Louhevaara VA, Smolander J, Alén MJ. Associations between work ability, healthrelated quality of life, physical activity and fitness among middle-aged men. Appl Ergon. 2007 (in press).

16. Sánchez P. Beneficios percibidos y adherencia a un programa de actividad física gerontológica. Revista Digital Educación Física y Deportes. 2002; 8: 52-5.

17. Anderson PJ, Critchley JA. Factor analysis of the metabolic syndrome: obesity vs. insulin resistance as the central abnormality. Int J Obes Relat Metab Disord 2001; 25: 1782-8.

18. Aschner C, Izquierdo J, Sole J, Tarazona A, Pinzón JB. Prevalence of the metabolic síndrome in a rural and urban population in Colombia. Diab Res Clin Pract 2002; 57 (suppl) 1: 532.

19. Sirit Y, Acero C, Bellorin M, Portillo R. Síndrome Metabólico y otros Factores de Riesgo Cardiovascular en Trabajadores de una Planta de Policloruro de Vinilo. Rev Salud Pública 2008; 10: 239-49.

20. Estrada J. Parámetros antropométricos de la población laboral colombiana 1995 (acopla95). Rev. Fac. Nac. Salud Pública 1998; 15:112-39.

21. López CA, Ramírez-Vélez R, Gallardo CEG, Marmolejo LC. Características morfofuncionales de individuos físicamente activos. Iatreia 2008; 21; 121-8.

22. Brook RD, Bard RL, Rubenfire M, Ridker PM, Rajagopalan S. Usefulness of visceral obesity (waist/hip ratio) in predicting vascular 
endothelial function in healthy overweight adults. Am J Cardiol 2001; 88: 1264-9.

23. Lugo LE, García HI, Gómez CR. Confiabilidad del cuestionario de calidad de vida en salud SF-36 en Medellín, Colombia. Rev Fac Nac Salud Pública 2006; 24: 37-50

24. ACSM American College Sport Medicine Metabolic Calculations. In: Guidelines for exercise testing and prescription. 2 ed. Philadelphia: Williams and Wilkins, 2000.

25. Tsai AC, Sandretto A, Chung YC. Dieting is more effective in reducing weight but exercise is more effective in reducing fat during the early phase of a weightreducing program in healthy humans. Journal of Nutritional Biochemistry 2003; 14:541-9

26 Cieza G, Stucki. Content comparison of healthrelated quality of life (HRQoL) instruments based on the international classification of functioning, disability, ad health (ICF). Qual Life Res 2005; 14: 1225-37.
27. Tuomi K, Ilmarinen J, Seitsamo J, Huuhtane P, Martikainen R, Nygård $\mathrm{CH}$, Klockars M. Summary of the Finnish Research Project (1981-1992) to promote the health and work ability of aging workers. Scand J Environ Health 1997;23:Suppl.1;66-71.

28. Ilmarinen J. Ageing Workers in the European Union-Status and Promotion of Work Ability, Employability and Employment 1999. Finnish Institute of Occupational Health, Ministry of Social Affairs and Health, Ministry of Labour, Helsinki.

29. Jansen N, Kant I, Van Amelswoort G, Kristensen T, Swaen G, Nijhuis F. Workfamily conflict as a risk factor for sickness absence. Occup Environ Med 2006; 6:48894.

30. Rodríguez F, Gusi N, Valenzuela A, Nàcher S, Nogués J, Marina M. Valoración de la condición física saludable en adultos (I): antecedentes y protocolos de la batería AFISAL-NEFC. Apunts Educación Física y Deportes 1998; 52: 54-75. 